\title{
INVARIANT INTEGRATION OVER THE INFINITE DIMENSIONAL ORTHOGONAL GROUP AND RELATED SPACES
}

\author{
BY \\ DAVID SHALE(1) \\ In Memory of Robert Mountjoy
}

1. Introduction. Integration over Hilbert space has been introduced by I. E. Segal and extensively developed by him and others. (See [2] and the bibliography cited there.) It now seems reasonable to enquire whether nonlinear manifolds of infinite dimension also possess integrals with appropriate mathematical properties. Indeed one of the proposals of Segal for quantum field theory [3] requires an integral over the space of solutions to a nonlinear hyperbolic partial differential equation. Recently the author, in collaboration with W.F. Stinespring, discovered an integral over the unit sphere of real Hilbert space which is invariant under the action of the orthogonal group. See [4]. This paper treats some examples of integration over closed manifolds of infinite dimension, that is, manifolds whose analogues of finite dimension are compact. Specifically we establish the existence, and the appropriate degree of uniqueness, of invariant integrals over the orthogonal group of a real Hilbert space, and unitary group of a complex Hilbert space, and some left coset spaces of these groups. As special cases we obtain a unitarily invariant integral over the pure states of a quantum mechanical system and the integral over the unit sphere of a real Hilbert space mentioned above. The aim has not been to do analysis over the spaces mentioned but rather to enquire how for simple concrete spaces an integral may actually be set up.

Our integrals are of the kind due to Segal and described in [2]. For each space we construct an integration algebra which is associated with it and which has the appropriate invariance properties.

Definition 1. An integration algebra $\tilde{\mathfrak{U}}$ is a pair $\{\mathfrak{A}, E\}$, where $\mathfrak{A}$ is a complex $a$-commutative *-algebra with unit and $E$ is a linear functional on $\mathfrak{U}$ such that the following properties hold: (1) $E(I)=1$. (2) $E\left(a^{*} a\right) \geqq 0$ for all $a$ in $\mathfrak{A}$ and if $E\left(a^{*} a\right)=0$ then $a=0$. (3) The following continuity property holds: For each $a$ in $\mathfrak{U}$ there is a constant $\mu_{a} \geqq 0$ such that $\left|E\left(b^{*} a\right)\right|^{2} \leqq \mu_{a}^{2} E\left(b^{*} b\right) E\left(c^{*} c\right)$ for all $b$ in $c$ in $\mathfrak{A}$. Any function $E$ with these properties will be called an expectation function on $\mathfrak{U}$.

Received by the editors October 22, 1965.

(1) Prepared with partial support from ONR contract Nonr-551(57). 
The following theorem due to Segal associates with each integration algebra an essentially unique probability measure space.

SEgal RePreSEntation THEOREM. If $\tilde{\mathfrak{U}}=\{\mathfrak{U}, E\}$ is an integration algebra, then there is a probability measure space $(M, \mathfrak{M}, m)$ and a representation $a \rightarrow a(x)$ of $\mathfrak{A}$ as bounded measurable functions (modulo null functions) on $M$, such that for each a in $\mathfrak{A}, E(a)=\int_{M} a(x) d m$. Further $\mathfrak{M}$ is the smallest $\sigma$-ring with regard to which all the functions $a(x)$ are measurable. Finally the measure ring of $\mathfrak{M}$, that is $\mathfrak{M}$ modulo the ideal of null sets, is uniquely determined by $\tilde{\mathfrak{A}}$.

Summary of Contents. The infinite dimensional spaces to be integrated on are approximated by classical left coset spaces. Facts concerning these together with useful notations are given in $\$ 3$. The construction of the integration algebras involves rather elaborate use of direct limits. The definitions and facts needed are collected in $\$ 2$. $\$ 4$ treats integration over the space of linear isometries which map a real Hilbert space of finite dimension into one of infinite dimension. $\S \S 5$ and 6 treat examples. In $\$ 7$ we construct the desired integration algebras over the infinite orthogonal and unitary groups.

2. Direct limits of integration algebras. The basic idea in integration over spaces of infinite dimension is to approximate them by spaces of finite dimension which themselves possess "natural" intgerals. Lifting the integrals from the different approximating spaces to the big space in a coherent way appears to require rather elaborate bookkeeping. We formulate this in terms of directed sets of integration algebras and of operations upon these directed sets. This section gathers together the necessary definitions and results.

Let $\tilde{\mathfrak{U}}=\{\mathfrak{U}, E\}$ be an integration algebra. Then $\mathfrak{A}$ with inner product $(a, b)=E\left(b^{*} a\right)$ is a prehilbert space. For each $a$ in $\mathfrak{A}$ the operator $L_{a}$ on $\mathfrak{A}$ defined by $L_{a} x=a x$ for all $x$ in $\mathfrak{A}$, extends to a bounded operator on the completion of $\mathfrak{A}$. If $\mu_{a}$ is as in Definition 1 , we have $\left\|L_{a}\right\| \leqq \mu_{a}$ and in fact $\mu_{a}$ may be taken to be $\left\|L_{a}\right\|$. Accordingly we introduce a norm on $\mathfrak{A}$ with $\|a\|$ being defined as $\left\|L_{a}\right\|$.

Let $\tilde{\mathfrak{U}}=\{\mathfrak{U}, E\}$ and $\tilde{\mathfrak{U}}^{\prime}=\left\{\mathfrak{U}^{\prime}, E^{\prime}\right\}$ be two integration algebras. By an algebraic isomorphism from $\tilde{\mathfrak{U}}$ to $\tilde{\mathfrak{U}}^{\prime}$ we merely mean an isomorphism from $\mathfrak{A}$ to $\mathfrak{U}^{\prime}$. However to define isomorphism we must add sufficient continuity conditions to ensure that if $\tilde{\mathfrak{U}}$ and $\tilde{\mathfrak{U}}^{\prime}$ are isomorphic then they yield equivalent probability measure spaces via the Segal Representation Theorem. Examination of the proof of this theorem shows that it is sufficient to define an isomorphism between $\tilde{\mathfrak{U}}$ and $\tilde{\mathfrak{U}}^{\prime}$ to be an algebraic isomorphism which preserves expectations and norms.

Definition 2. A directed set of integration algebras is a triple $\left\{\Lambda, \tilde{\mathfrak{A}}_{\alpha}, \pi_{\beta, \alpha}^{*}\right\}$ where $\Lambda$ is a directed set; for each $\alpha$ in $\Lambda, \tilde{\mathfrak{A}}_{\alpha}$ is an integration algebra; and $\pi_{\beta, \alpha}^{*}$ which is defined for $\alpha$ and $\beta$ in $\Lambda$ with $\alpha \leqq \beta$, is an isomorphism of $\tilde{\mathfrak{A}}_{\alpha}$ into $\tilde{\mathfrak{A}}_{\beta}$. It is always supposed further that if $\alpha \leqq \beta \leqq \gamma$, then $\pi_{\gamma, \alpha}^{*}=\pi_{\gamma, \beta}^{*} \pi_{\beta, \alpha}^{*}$. 
We use the notation $\pi_{\beta, \alpha}^{*}$ because in applications $\pi_{\beta, \alpha}$ is always the dual of a mapping on sets.

DEFINITION 3. Let $\boldsymbol{F}=\left\{\Lambda, \tilde{\mathfrak{I}}_{\alpha}, \pi_{\beta, \alpha}^{*}\right\}$ be a directed set of integration algebras. A direct limit of $\boldsymbol{F}$ is a pair $\left\{\tilde{\mathfrak{U}}, \eta_{\alpha}^{*}\right\}$ where $\tilde{\mathfrak{U}}$ is an integration algebra and for each $\alpha$ in $\Lambda, \eta_{\alpha}^{*}$ is an isomorphism of $\tilde{\mathfrak{A}}_{\alpha}$ into $\tilde{\mathfrak{U}}$; provided the following conditions are satisfied: (i) $\mathfrak{A}=U \eta_{\alpha}^{*}\left(\mathfrak{A}_{\alpha}\right)$ where the union is over $\Lambda$; (ii) $\eta_{\beta}^{*} \pi_{\beta, \alpha}^{*}=\eta_{\alpha}^{*}$ for all $\alpha$ and $\beta$ in $\Lambda$ with $\alpha \leqq \beta$.

The following proposition is essentially the same as Theorem 1 in [1]. The proof is omitted.

Proposition 1. Any directed set of integration algebras has a direct limit which is unique up to isomorphism.

A subset $\Lambda_{0}$ of a directed set $\Lambda$ will be called admissible if it is nonempty and if it contains $\alpha$ then it contains all $\beta$ in $\Lambda$ with $\beta \geqq \alpha$. Let $\boldsymbol{F}=\left\{\Lambda, \tilde{\mathfrak{U}}_{\alpha}, \pi_{\beta, \alpha}^{*}\right\}$ be a directed set of integration algebras. It is clear that if $\Lambda_{0}$ is an admissible subset of $\Lambda$ and if $\boldsymbol{F}^{\prime}=\left\{\Lambda_{0}, \tilde{\mathfrak{A}}_{\alpha}, \pi_{\beta, \alpha}^{*}\right\}$, where $\alpha$ and $\beta$ are restricted to $\Lambda_{0}$, then the direct limit of $\boldsymbol{F}$ may be naturally identified with that of $\boldsymbol{F}^{\prime}$. Accordingly we give:

Definition 4. Let $F=\left\{\Lambda, \tilde{\mathfrak{U}}_{\alpha}, \pi_{\beta, \alpha}^{*}\right\}$ and $\boldsymbol{F}^{\prime}=\left\{\Lambda^{\prime}, \tilde{\mathfrak{U}}_{\alpha}, \pi_{\beta, \alpha}^{* \prime}\right\}$ be two directed sets of integration algebras. An isomorphism $\Gamma$ from $\boldsymbol{F}$ to $\boldsymbol{F}^{\prime}$ is a pair $\left\{\lambda, \Phi_{\alpha}\right\}$ where $\lambda$ is a 1-1 map of an admissable subset $\Lambda_{0}$ of $\Lambda$ onto an admissible subset of $\Lambda^{\prime}$ which preserves the order relations, and where for each $\alpha$ in $\Lambda_{0}, \Phi_{\alpha}$ is an isomorphism from $\tilde{\mathfrak{I}}_{\alpha}$ to $\mathfrak{I}_{\lambda_{\alpha}}^{\prime}$. It is always supposed that if $\alpha$ and $\beta$ are in $\Lambda_{0}$ and $\alpha \leqq \beta$ then $\pi_{\lambda, \beta, \lambda \alpha}^{*} \Phi_{\alpha}=\Phi_{\beta} \pi_{\beta, \alpha}^{*}$.

Since the intersection of two admissable subsets of $\Lambda$ is again admissible there is no loss in identifying two isomorphisms between $\boldsymbol{F}$ and $\boldsymbol{F}^{\prime}$ if they agree on an admissible subset of $\Lambda$ and in what follows this is always done.

Now let $\boldsymbol{F}^{\prime \prime}=\left\{\Lambda^{\prime \prime}, \tilde{\mathfrak{X}}_{\alpha}^{\prime \prime} \pi_{\beta, \alpha}^{* \prime \prime}\right\}$ be a third directed set of integration algebras. Let $\Gamma^{\prime}=\left\{\lambda^{\prime}, \Phi_{\alpha}^{\prime}\right\}$ be an isomorphism from $\boldsymbol{F}^{\prime}$ to $\boldsymbol{F}^{\prime \prime}$ defined on an admissible subset $\Lambda_{0}^{\prime}$ of $\Lambda^{\prime}$. We define $\Gamma^{\prime} \Gamma$ to be $\left\{\lambda^{\prime} \lambda, \Phi_{\lambda \alpha}^{\prime} \Phi_{\alpha}\right\}$ where $\alpha$ ranges over the admissible set of those elements in $\Lambda$ whichare mapped into $\Lambda_{0}^{\prime}$ by $\lambda$.

Proposition 2. (a) Let $\boldsymbol{F}=\left\{\Lambda, \tilde{\mathfrak{I}}_{\alpha}, \pi_{\beta, \alpha}^{*}\right\}$ and $\boldsymbol{F}^{\prime}=\left\{\Lambda^{\prime}, \tilde{\mathfrak{I}}_{\alpha}, \pi_{\beta, \alpha}^{* \prime}\right\}$ be directed sets of integration algebras. Let $\left\{\tilde{\mathfrak{U}}, \eta_{\alpha}^{*}\right\}$ and $\left\{\tilde{\mathfrak{I}}^{\prime}, \eta_{\alpha}^{* \prime}\right\}$ be the corresponding direct limits. Suppose that $\Gamma=\left\{\lambda, \Phi_{\alpha}\right\}$ is an isomorphism from $\boldsymbol{F}$ to $\boldsymbol{F}^{\prime}$. Then there is a unique isomorphism $\Phi_{\Gamma}$ from $\tilde{\mathfrak{I}}$ to $\tilde{\mathfrak{U}}^{\prime}$ such that $\Phi_{\Gamma} \eta_{\alpha}^{*}=\eta_{\lambda \alpha}^{* \prime} \Phi_{\alpha}$ whenever the right-side is defined.

(b) If $\boldsymbol{F}^{\prime \prime}$ is a third directed set of integration algebras and $\Gamma^{\prime}$ is an isomorphism from $\boldsymbol{F}^{\prime}$ to $\boldsymbol{F}^{\prime \prime}$, then $\Gamma^{\prime} \Gamma$ is an isomorphism from $\boldsymbol{F}$ to $\boldsymbol{F}^{\prime \prime}$ and $\Phi_{\Gamma^{\prime} \Gamma}=\Phi_{\Gamma^{\prime}} \Phi_{\Gamma}$

Proofs are omitted.

The integration algebras we wish to construct should have a group of sym- 
metries associated with them. However the desired groups do not act, in the first instance, upon the final integration algebras but rather upon the directed sets of integration algebras used in their construction. The following definition and result were designed to take care of this situation.

Definition 5. Let $\boldsymbol{F}=\left\{\Lambda, \tilde{\mathfrak{A}}_{\alpha}, \pi_{\beta, \alpha}^{*}\right\}$ be a directed set of integration algebras. Let $G$ be a group with unit element $e$. Suppose that $G$ has a representation as order preserving permutations of $\Lambda$, the action of $g$ in $G$ on $\alpha$ in $\Lambda$ being denoted by $g \alpha$. Suppose further that for each $g$ in $G$ and each $\alpha$ in $\Lambda$ there is an isomorphism $\omega_{\alpha}(g)$ of $\tilde{\mathfrak{H}}_{\alpha}$ onto $\tilde{\mathfrak{A}}_{g \alpha}$, such that the following conditions are satisfied: (i) For each $\alpha$ in $\Lambda \omega_{\alpha}(e)$ is the identity isomorphism on $\tilde{\mathfrak{A}}_{\alpha}$. (ii) For all $\alpha$ in $\Lambda$ and all $g$ and $g_{1}$ in $G$ we have $\omega_{g x}\left(g_{1}\right) \omega_{\alpha}(g)=\omega_{\alpha}\left(g_{1} g\right)$. (iii) If $\alpha$ and $\beta$ are in $\Lambda$ and $\alpha \leqq \beta$ then $\omega_{\beta}(g) \pi_{\beta, \alpha}^{*}=\pi_{g \beta, g \alpha}^{*} \omega_{\alpha}(g)$. Then $G$ will be called a symmetry group of $\boldsymbol{F}$.

The subgroup $G_{\alpha}$ of $G$ consisting of those elements which leave $\alpha$ fixed, will be called the stability group of of $\alpha$. Then $\omega_{x}\left({ }^{\circ}\right)$ restricted to $G_{\alpha}$ is a group representation of $G_{\alpha}$ as automorphisms of $\tilde{\mathfrak{U}}_{\alpha}$.

Proposition 3. (a) Let $\boldsymbol{F}=\left\{\Lambda, \tilde{\mathfrak{I}}_{x}, \pi_{\beta \alpha}^{*}\right\}$ be a directed set of integration algebras with direct limit $\left\{\tilde{\mathfrak{U}}, \eta_{x}^{*}\right\}$. Let $G$ be a symmetry group of $\boldsymbol{F}$ as defined above. Then there is a unique representation $W(\cdot)$ of $G$ as automorphisms of $\tilde{\mathfrak{U}}$ such that $W(g) \eta_{x}^{*}=\eta_{g \alpha}^{*} \omega_{\alpha}(g)$ for all $\alpha$ in $\Lambda$ and all $g$ in $G$. (b) Now suppose that $\tilde{\mathfrak{U}}_{\alpha}=\left\{\mathfrak{U}_{\alpha}, E_{\alpha}\right\}$ and that $\tilde{\mathfrak{U}}=\{\mathfrak{A}, E\}$. Suppose that for each $\alpha$ in $\Lambda, E_{\alpha}$ is the only expectation function on $\mathfrak{A}_{\alpha}$ which is invariant under the representation $\omega_{\alpha}(\cdot)$ of $G_{\alpha}$ the stability group of $\alpha$. Then $E$ is the only expectation function on $\mathfrak{A}$ that is invariant under the representation $W(\cdot)$ of $G$.

Proofs are omitted.

3. Left coset spaces. Let $K$ and $H$ be real Hilbert spaces. Suppose that $K$ has finite dimension and that $\operatorname{dim}(K) \leqq \operatorname{dim}(H)$. Then $\theta(K, H)$ will always denote the collection of linear isometries which map $K$ into $H$ and $O(K)$ and $O(H)$ will denote the orthogonal groups on $K$ and $H$ respectively. The orthogonal groups $O(H)$ and $O(K)$ act on $\theta(K, H)$ by left and right multiplication respectively. There is no loss in supposing that $K$ is a subspace of $H$. If $H^{\prime}$ is the orthogonal complement of $K$ in $H$, then $\theta(K, H)$ may be identified in a natural way with the space of left cosets $O(H) / O\left(H^{\prime}\right)$. We simply identify $T$ in $\theta(K, H)$ with the collection of operators in $O(H)$ which agree with it on $K$.

Now suppose that $H$ has finite dimension. Then it is well known that $O(H) / O\left(H^{\prime}\right)$ and hence also $\theta(K, H)$, admits a unique regular probability measure invariant under the action of $O(H)$. The algebra of bounded Borel functions on $\theta(K, H)$ will always be denoted by $\mathfrak{A}_{K, H}$ and the invariant expectation corresponding to the measure by $E_{K, H}$. Then $\tilde{\mathfrak{U}}_{K, H}=\left\{\mathfrak{U}_{K, H}, E_{K, H}\right\}$ is an integration algebra. The action of $O(H)$ on $\mathfrak{I}_{K, H}$ is given for any $S$ in $O(H)$, by $L_{s}$ where $L_{s} f(T)$ $=f\left(S^{-1} T\right)$. We can rephrase the uniqueness of the probability measure on $\theta(K, H)$ 
by saying that $E_{K, H}$ is the unique expectation function on $\mathfrak{A}_{K, H}$ that is invariant under the representation $L_{(.)}$of $O(H)$.

The following rather technical result will be used in $\$ \S 4$ and 7.

Proposition 5. Let $K, H, L$ and $M$ be Hilbert spaces with $K \subseteq L, H \subseteq M$, $\operatorname{dim}(K) \leqq \operatorname{dim}(H)<\infty$ and $\operatorname{dim}(L) \leqq \operatorname{dim}(M)<\infty$. Let $G$ be the subgroup of elements in $O(M)$ which leave $H$ invariant. For $T$ in $G$, let $T^{\prime}$ be the restriction of $T$ to $H$. Let $\theta^{\prime}$ be an open subset of $\theta(L, M)$ which is invariant under $G$ and whose complement in $\theta(L, M)$ is a null set. Let $\pi$ be a continuous map from $0^{\prime}$ onto $\theta(K, H)$ such that $T^{\prime} \pi=\pi T$ for all $T$ in $G$. Then the dual map $\pi^{*}$ determines an isomorphism of $\tilde{\mathfrak{A}}_{K, H}$ into $\tilde{\mathfrak{U}}_{L, M}$.

Proof. For $f$ in $\mathfrak{I}_{K, H}, \pi^{*} f(T)=f(\pi T)$. We may consider the regular measure determined by $E_{L, M}$ to be on $\theta^{\prime}$ instead of on $\theta(L, M)$. It is then clear that $\pi^{*}$ determines an algebraic isomorphism from $\mathfrak{A}_{K, H}$ to $\mathfrak{U}_{L, M}$ and further that ess. sup $|f|=$ ess. sup $\left|\pi^{*} f\right|$, for any $f$ in $\mathfrak{A}_{I, K}$. But for $g$ in either $\mathfrak{A}_{K, H}$ or in $\mathfrak{A}_{L, M}$ ' ess. sup $|g|=\|g\|$. Therefore $\|f\|=\left\|\pi^{*} f\right\|$. It remains to show that $\pi^{*}$ preserves expectations.

Consider the expectation function $E^{\prime}$ on $\mathfrak{A}_{K, H}$ given by $E^{\prime}(f)=E_{L, M}\left(\pi^{*} f\right)$. Since for $T$ in $G, \pi T=T^{\prime} \pi$, it follows that $E^{\prime}$ is invariant under the action of $O(H)$. Therefore $E^{\prime}=E_{K, H}$ and $\pi^{*}$ preserves expectations.

4. Invariant integration over the spaces $\theta(K, H)$. We suppose that $H$ has infinite dimension. Let $\Lambda_{K}$ be the directed set of finite dimensional subspaces of $M$ of $H$ with $\operatorname{dim}(M) \geqq \operatorname{dim}(K)$ ordered by inclusion. To each $M$ in $\Lambda_{K}$ we associate the integration algebra $\tilde{\mathfrak{A}}_{K, M}$ that was defined in $\S 3$. We now define mappings $\pi_{K, M, N}$ from $\theta(K, N)$ to $\theta(K, M)$ with duals $\pi_{K, N, M}^{*}$ so that $\boldsymbol{F}_{\boldsymbol{K}}$ $=\left(\Lambda_{K}, \widetilde{\mathfrak{A}}_{K, M}, \pi_{K, N, M}^{*}\right)$ is a directed set of integration algebras.

To this end let $e_{1}, \cdots, e_{k}$ be a fixed orthonormal basis for $K$. To specify $T$ in $\theta(K, H)$ it is sufficient to give the sequence $T e_{1}, \cdots, T e_{k}$. Let $N$ be any subspace of $H$ with $M \subseteq N \subseteq H$. Let $P$ be the projection of $N$ on $M$. Finally if $x_{1}, \cdots, x_{k}$ is any sequence of vectors, let $\boldsymbol{G}\left\{x_{1}, \cdots, x_{k}\right\}$ be the sequence obtained by applying the Gram-Schmidt orthogonalization process. Then for $T$ in $\theta(K, N)$ we define $\pi_{K, M, N} T$ to be the map which sends $e_{1}, \cdots, e_{k}$ to $G\left\{P T e_{1}, \cdots, P T e_{k}\right\}$.

We first show that $\theta(K, M), \theta(K, N)$ and $\pi_{K, M, N}$ satisfy the hypotheses of Proposition 4. Let $d_{1}, \cdots, d_{n}$ be an orthonormal basis for $N$ chosen so that the subsequence $d_{1}, \cdots, d_{m}$ spans $M$. There is no loss in supposing that $m<n$. Let $t_{i, j}=\left(T e_{i}, d_{j}\right)$. Let $T$ be matrix $\left\{t_{i, j}\right\}$ with $1 \leqq i \leqq k$ and $1 \leqq j \leqq n$. Let $\boldsymbol{T}^{\prime}$ be the submatrix $\left\{t_{i, j}\right\}$ with $1 \leqq i \leqq k$ and $1 \leqq j \leqq m$. That is, $\boldsymbol{T}^{\prime}$ is just $\boldsymbol{T}$ with the last $n-m$ columns deleted. Then the $i$ th row of $\boldsymbol{T}$ gives the coefficients of the expansion of $T e_{i}$ with regard to the basis $d_{1}, \cdots, d_{n}$ of $N$ while the $i$ th row of $T^{\prime}$ gives the analogous coefficients for $P T e_{i}$ with regard to the basis $d_{1}, \cdots, d_{m}$ of $M$. Now $\pi_{K, M, N} T$ is defined if and only if $T^{\prime}$ has rank $k$, that is 
if and only if at least one $k \times k$ minor of $\boldsymbol{T}^{\prime}$ has a nonzero determinant. The subset of $\theta(K, M)$ defined by the vanishing of one of these determinants is a submanifold of lower dimension and hence a closed null set of $\theta(K, N)$.

Let $\theta^{\prime}$ be the set where $\pi_{K, M, N}$ is defined. Then the complement of $\theta^{\prime}$ in $\theta(K, N)$ is a finite union of such submanifolds and consequently is a null set.

The Gram-Schmidt process $\boldsymbol{G}$ may be considered as acting on the open set of matrices of rank $k$. Clearly $G$ is continuous. It follows that $\pi_{K, M, N}$ is continuous on $\theta^{\prime}$.

Let $G$ be the subgroup of $O(N)$ which leaves the subspace $M$ invariant. Any orthogonal operator $S$ in $G$ commutes with $P$ the projection of $N$ on $M$. It follows that $\left(P T e_{1}, \cdots, P T e_{k}\right)$ and $\left(P S T e_{1}, \cdots, P S T e_{k}\right)$ span spaces of the same dimension. Hence $\theta^{\prime}$ is invariant under $G$.

If $\left\{x_{1}, \cdots, x_{n}\right\}$ is a $k$-tuple of vectors in $N$ and $A$ is a linear operator on $N$ then $A\left\{x_{1}, \cdots, x_{k}\right\}$ will denote the $k$-tuple $\left\{A x_{1}, \cdots, A x_{k}\right\}$. If $x_{1}, \cdots, x_{k}$ are linearly independent and $S$ is an orthogonal operator we have $\boldsymbol{G} S\left\{x_{1}, \cdots, x_{k}\right\}=S G\left\{x_{1}, \cdots, x_{k},\right\}$. It follows that if $T$ is in $\theta^{\prime}$ and $S$ is in $G$ then

$$
S G\left\{P T e_{1}, \cdots, P T e_{k}\right\}=\boldsymbol{G}\left\{P S T e_{1}, \cdots, T e_{k}\right\} .
$$

This shows that $S$ in $G$ commutes with $\pi_{K, M, N}$.

We have established all the conditions of Proposition 4. Consequently this proposition implies that $\pi_{K, N, M}^{*}$ is an isomorphism of $\tilde{\mathfrak{U}}_{K, M}$ into $\tilde{\mathfrak{A}}_{K, N}$.

To establish that $\boldsymbol{F}_{K}=\left\{\Lambda_{K}, \tilde{\mathfrak{A}}_{K, M}, \pi_{K, N, M}^{*}\right\}$ is a directed set of integration algebras it remains only to show that $\pi_{K, M, N} \pi_{K, N, L}=\pi_{K, M, L}$, where $M \subseteq N \subseteq L$. This in turn devolves into showing that if $x_{1}, \cdots, x_{k}$ are linearly independent vectors in $N$, with $P x_{1}, \cdots, P x_{k}$ also linearly independent, then

$$
\boldsymbol{G P}\left\{x_{1}, \cdots, x_{k}\right\}=\boldsymbol{G} \boldsymbol{P} \boldsymbol{G}\left\{x_{1}, \cdots, x_{k}\right\} .
$$

We establish this by induction. If $k=1$ it is trivial. Assume therefore that the result is true for $k=r$. Let $k=r+1$. It is enough to show that $\boldsymbol{G P}\left\{x_{1}, \cdots, x_{r}, x_{r+1}\right\}$ and $P G P\left\{x_{1}, \cdots, x_{r}, x_{r+1}\right\}$ have the same last entry. Let $Q$ be the projection of $N$ on the linear span of $x_{1}, \cdots, x_{r}$ and $Q^{\prime}$ to be the projection of $M$ on the linear span of $P x_{1}, \cdots, P x_{r}$. It is enough to show that $(I-Q) P=(I-Q) P\left(I-Q^{\prime}\right)$ or equivalently that $(I-Q) P Q^{\prime}=0$. The latter follows since $P Q^{\prime} N$ is the subspace of $M$ spanned by $P x_{1}, \cdots, P x_{r}$, while $(I-Q)$ is the projection of $M$ into the orthogonal complement of this subspace.

We can now define the desired integration algebra over $\theta(K, H)$. This is just $\left\{\tilde{\mathfrak{A}}_{K}, \eta_{K, M}^{*}\right\}$ the direct limit of $\boldsymbol{F}_{K}=\left\{\Lambda_{K}, \tilde{\mathfrak{A}}_{K, M}, \pi_{K, N, M}^{*}\right\}$. Let $\tilde{\mathfrak{A}}_{K}=\left\{\tilde{\mathfrak{A}}_{K}, E_{K}\right\}$. Then $\mathfrak{U}_{K}$ can be interpreted as an algebra of functions on $\theta(K, H)$. To do this we define $\pi_{K, M, H}$ analogously to $\pi_{K, M, N}$ and set $\eta_{K, M}^{*}=\pi_{K, H, M}^{*}$. Then $\mathfrak{A}_{K}$ may be identified with $U_{M} \eta_{K, M}^{*}\left(\mathfrak{U}_{K, M}\right)$. It should be noted however that every element of $\mathfrak{U}_{K}$ is singular and what the null functions are is obscure. 
The orthogonal group $O(H)$ is a symmetry group of $\boldsymbol{F}_{K}$. The natural action of $S$ in $O(H)$ is that it sends $M$ in $\Lambda_{K}$ to $S M ; T$ in $\theta(K, M)$ to $S T$ in $\theta(K, S M)$; and $f(T)$ on $\theta(K, M)$ to $L_{S} f(T)$ on $\theta(K, S M)$, where $L_{S} f(T)=f\left(S^{-1} T\right)$. It is straightforward to check that the action of $S$ commutes with the maps $\pi_{K, M, N}$. Hence by part (a) of Proposition 3, the action of $O(H)$ on $\mathfrak{A}_{K}$, which is again given by $L_{S} f(T)=f\left(S^{-1} T\right)$, preserves the expectation $E_{K}$. Upon considering the action of $O(H)$ on $F_{K}$ we see that the stability group of $M$ in $\Lambda_{K}$ is just $O(M) \oplus O\left(M^{\perp}\right)$. Now $E_{K, M}$ is the only expectation on $\mathfrak{A}_{K}$ that is invariant under the action $L_{S}$ of $O(M)$. It follows by part (b) of Proposition 3 that $E_{K}$ is the only expectation function on $\mathfrak{I}_{K}$ that is invariant under the action $L_{S}$ of $O(H)$. Finally we remove the dependence of the theory on a special basis $e_{1}, \cdots, e_{k}$ for $K$ by showing that $E_{K}$ is invariant under the action-on-the-right of $O(K)$. Let $S$ be in $O(K)$. Then $S$ acts on $\theta(K, M)$ by $T \longrightarrow T S$ and on $\mathfrak{A}_{K, M}$ by $f(T) \rightarrow R_{S} f(T)$ where $R_{S} f(T)=f(T S)$. The automorphism $R_{S}$ extends to an algebraic automorphism of $\tilde{\mathfrak{A}}_{K}$. For $f(T)$ in $\mathfrak{\mathfrak { U }}_{K}$ let $E^{\prime}(f)=E_{K}\left(R_{S} f\right)$. Then $E^{\prime}$ is an expectation function on $\mathfrak{A}_{K}$ which is invariant under $O(H)$. Therefore $E^{\prime}=E_{K}$.

We have established the following result:

Theorem 1. Let $K$ and $H$ be real Hilbert spaces. Suppose that $K$ and $H$ have finite and infinite dimensions respectively. Let $\theta(K, H)$ be the space of linear isometries mapping $K$ into $H$. Let $\mathfrak{I}_{K}$ be the algebra over $\theta(K, H)$ that was constructed above. Then $\mathfrak{I}_{K}$ has a unique expectation $E_{K}$ which is invariant under the action $L_{(.)}$of $O(H)$ on $\mathfrak{P}_{K}$ where $L_{S} f(T)=f\left(S^{-1} T\right)$. The expectation $E_{K}$ is then invariant under the action $R_{(.)}$of $O(K)$ on $\mathfrak{U}_{K}$ where $R_{S} f(T)=f(T S)$.

5. Invariant integration over the unit sphere of a real Hilbert space. Let $H$ be a real Hilbert space with infinite dimension. Let $S=\{x:\|x\|=1\}$ be the unit sphere in $H$. If $K$ is a real Hilbert space of dimension 1 and $e$ is one of the unit vectors in $K$, then the map on $\theta(K, H)$ sending $T$ to $T e$ identifies $\theta(K, H)$ with $S$.

Let $M$ be any subspace of $H$ with finite positive dimension. Let $P$ be the projection of $H$ on $M$. Set $S_{M}$ be $S \cap M$ and $\Phi$ be any bounded Borel function on $M$. Then a function $f$ on $S$ of the form $f(x)=\Phi\left(\|P x\|^{-1} P x\right)$ will be called tame. Letting both $M$ and $\Phi$ vary, the collection of tame functions obtained form an algebra $\mathfrak{A}_{s}$. This is just the algebra $\mathfrak{U}_{K}$ of $\S 4$. Further for $f$ in $\mathfrak{A}_{s}$ the corresponding expectation is $E_{S}(f)=\int \Phi d m$ where the integral is taken over $S_{M}$ and $d m$ is the element of surface area normalised so that $S_{M}$ has integral 1. Thus the integration algebra $\left\{\mathfrak{U}_{S}, E_{S}\right\}$ over $S$ is one given in $\S 4$.

A function $f$ on $H$ itself is called tame if $f(x)==\Psi(P x)$ where $\Psi$ is a bounded Borel function on $M$. Let $\mathfrak{I}_{I}$ be the algebra formed by all the tame functions on $H$. For $f$ in $\mathfrak{X}_{H}$, let $E_{H}(f)=\int_{M} \Psi d \eta$ where $d \eta$ is the Gaussian measure $(2 \pi c)^{-n / 2} \exp (-(x, x) / 2 c) d_{n} x$ with $n=\operatorname{dim}(M)$. The pair $\left\{\mathfrak{A}_{H}, E_{H}\right\}$ is the nor- 
mal distribution on $H$ as described in [2]. We identify $S$ with the rays in $H$ going outwards from the origin. The algebra $\mathfrak{A}_{S}$ then becomes identified with the subalgebra of $\mathfrak{U}_{H}$ consisting of those tame functions which are positively homogeneous of degree zero. We have further that $E_{S}=E_{H}$ on $\mathfrak{U}_{\boldsymbol{S}}$.

Finally we remark that if $A$ is a self-adjoint operator on $H$ the function $f(x)=(A x, x)$ on $S$ cannot be interpreted as a measurable functional over $S$ unless $A$ is constant. This is established in [4].

6. Invariant integration over the pure states of a quantum mechanical system. Let $H$ and $K$ be complex Hilbert spaces. Suppose that $K$ has finite nonzero dimension and that $H$ has infinite dimension. Then $\theta(K, H)$ will denote the collection of complex-linear isometries which map $K$ into $H$. With obvious changes, the arguments of $\S 4$ yield a unitarily invariant integral over $\theta(K, H)$. When $K$ has dimension 1 we get an invariant integral over the unit sphere $\boldsymbol{S}$ of $H$. If $(x, y)$ is the inner product in $H$, let $[x, y]=\operatorname{Real}(x, y)$. Let $H_{R}$ be $H$ with real operations only and inner product $[x, y]$. Then $S$ is also the unit sphere in $H_{R}$ and the integration algebra $\left\{\mathfrak{U}_{S}, E_{S}\right\}$ over $S$ is exactly as in $\S 5$.

The pure states of a quantum mechanical system may be identified with the set of unitary rays over a complex Hilbert space. Specifically if $H$ is the complex Hilbert space and $\boldsymbol{P}$ is the collection of pure states, then an element $\boldsymbol{P}$ is a set $\{\lambda x$ such that $|\lambda|=1\}$ where $x$ is a unit vector in $H$. Let $\Lambda$ be the orthogonal operator on $H_{R}$ corresponding to $i I$ on $H$. Then any $f$ in $\mathfrak{A}_{S}$ will be said to be based on $\boldsymbol{P}$ if $f(\exp (\Lambda t) x)=f(x)$ for all real $t$ and all $x$ in $H$. Let $\mathfrak{A}_{\boldsymbol{P}}$ be the subalgebra of functions in $\mathfrak{U}_{S}$ which are based on $\boldsymbol{P}$. Let $E_{\boldsymbol{P}}$ be $E_{\boldsymbol{S}}$ restricted to $\mathfrak{A}_{\boldsymbol{P}}$. Then $\left\{\mathfrak{U}_{\boldsymbol{p}}, E_{\boldsymbol{P}}\right\}$ is the desired unitarily invariant integration algebra over $\boldsymbol{P}$

The analogue of the remark at the end of the last section is that if $A$ is an observable of the quantum mechanical system, that is a self-adjoint operator on $H$, then the function that assigns to each pure state $s$ the expectation of $A$ in $s$ cannot be measurable, let alone integrable, unless $A$ is a constant. However if $A$ leaves a finite dimensional subspace $M$ of $H$ invariant and equals zero on $M^{\perp}$, and $\boldsymbol{P}$ is the projection on $M$, then $(A x, x) /(P x, x)$ does yield an integrable function on $\boldsymbol{P}$.

7. Invariant integration over the orthogonal group $O(H)$. We remark, that with obvious changes, the arguments of this section apply equally to the unitary group on a complex Hilbert space.

Let $H$ be a real Hilbert space of infinite dimension. Let $\Lambda$ be the directed set formed by the nontrivial subspaces of $H$ with finite dimension. Here again inclusion gives the partial ordering. To each $K$ in $\Lambda$ we associate the integration algebra $\widetilde{\mathfrak{U}}_{K}=\left\{\mathfrak{U}_{K}, E_{K}\right\}$ that was constructed over $\theta(K, H)$ in $\$ 4$. We proceed to construct maps $\Phi_{L, K}^{*}$ so that $F=\left\{\Lambda, \tilde{\mathfrak{A}}_{K}, \Phi_{L, K}^{*}\right\}$ is a directed set of integration algebras.

Suppose that $K$ and $L$ are subspaces in $\Lambda$ with $K \leqq L$ and that $M$ is a subspace 
with $\operatorname{dim}(L) \leqq \operatorname{dim}(M)<\infty$. Let $\Phi_{M, K, L}$ be the map from $\theta(L, M)$ to $\theta(K, M)$ which sends $T$ to its restriction to $K$. Proposition 4 implies that the dual map $\Phi_{M, L, K}^{*}$ is an isomorphism from $\tilde{\mathfrak{U}}_{K, M}$ into $\tilde{\mathfrak{A}}_{L, M}$.

Suppose that $e_{1}, \cdots, e_{r}$ is an orthonormal basis for $L$ chosen so that $e_{1}, e_{2}, \cdots, e_{k}$ is an orthonormal basis for $K$. Let $\boldsymbol{F}_{K}=\left\{\Lambda_{K}, \tilde{\mathfrak{A}}_{K, M}, \pi_{K, N, M}^{*}\right\}$ and $F_{L}=\left\{\Lambda_{L}, \tilde{\mathfrak{A}}_{L, M}, \pi_{L, N, M}^{*}\right\}$ be the directed sets of integration algebras constructed with regard to these bases as described in $\S 4$. Then the maps $\Phi_{M, L, K}^{*}$ determine an isomorphism from $\boldsymbol{F}_{K}$ to $\boldsymbol{F}_{L}$ in the sense of Definition 4. To see this let $K$, $L$ and $M$ be as above and let $N$ be a subspace with $\operatorname{dim}(M) \leqq \operatorname{dim}(N)<\infty$. It is enough to show that $\pi_{L, N, M}^{*} \Phi_{M, L, K}^{*}=\Phi_{N, L, K}^{*} \pi_{K, N, M}^{*}$ or equivalently that $\pi_{K, M, N} \Phi_{N, K, L}=\Phi_{M, K, L} \pi_{L, M, N}$ off the subset of $\theta(L, N)$ where $\pi_{L, M, N}$ is not defined. Suppose that $T$ in $\theta(L, N)$ does not belong to this set. Let $P$ be the projection of $N$ on $M$ and $\boldsymbol{G}$ denote the Gram-Schmidt process. Then both sides of the above equation transform $T$ to the map which sends $e_{1}, \cdots, e_{k}$ to $G\left\{P T e_{1}, \cdots, P T e_{k}\right\}$.

We may now define $\Phi_{L, K}^{*}$ to be the isomorphism from $\tilde{\mathfrak{A}}_{K}$ to $\tilde{\mathfrak{A}}_{L}$ which is determined by the isomorphism from $\boldsymbol{F}_{K}$ to $\boldsymbol{F}_{L}$ described above. It is clear that $\Phi_{L, K}^{*}$ does not depend on the basis $e_{1}, \cdots, e_{r}$. Let $F$ be $\left\{\Lambda, \tilde{\mathfrak{A}}_{K}, \Phi_{L, K}^{*}\right\}$. It may be seen from part (b) of Proposition 2 that $\boldsymbol{F}$ is a directed set of integration algebras. Let $\left\{\tilde{\mathfrak{A}}^{*}, \eta_{K}\right\}$ be the direct limit of $\boldsymbol{F}$. Let $\tilde{\mathfrak{A}}$ be $\{\mathfrak{A}, E\}$. We realize $\tilde{\mathfrak{A}}$ as an algebra of function classes as follows: Let $\Phi_{K}$ be the map from $O(H)$ to $\theta(K, H)$ which sends $T$ into its restriction to $K$. Let $\Phi_{K}$ be the dual map from $\mathfrak{A}_{K}$ to function classes on $O(H)$. Then $\eta_{K}^{*}$ may be taken to be $\Phi_{K}^{*}$ and $\mathfrak{A}$ may be taken to be $U \Phi_{K}\left(\mathfrak{A}_{K}^{*}\right)$, where the union is taken over subspaces of $H$ having finite positive dimension. It is not directly apparent, to the author at least, that $\mathfrak{A}$ defined in this way is even an algebra of function classes. Hence the circuitous definition.

Recall that for $f(T)$ in $\mathfrak{A}_{K}$ and $S$ in $O(H), L_{S} f(T)=f\left(S^{-1} T\right)$. The automorphisms $L_{(\cdot)}$ also denote the induced action on the direct limit $F$. It follows from part (a) of Proposition 3 that $E$ is invariant under the action of $L_{(\cdot)}$ on $\mathfrak{A}$. Further, Theorem 1 and part (b) of Proposition 3 imply that $E$ is the unique expectation on with this invariance property.

Let $S$ be in $O(H)$. The natural action-on-the-right of $S$ sends $K$ in $\Lambda$ to $S K$, and $\mathfrak{A}_{K}$ to $\mathfrak{A}_{S^{-1} \mathbb{K}_{K}}$ via $R_{S} f(T)=f(T S)$. It may be seen that these maps determine an isomorphism of $\boldsymbol{F}=\left\{\Lambda, \tilde{\mathfrak{A}}_{K}, \Phi_{L, K}^{*}\right\}$ and hence an isomorphism of $\mathfrak{\mathfrak { A }}$ itself.

We have established the following result.

THEOREM 2. Let $O(H)$ be the group of orthogonal operators on the infinite dimensional real Hilbert space $H$. Let $\mathfrak{A}$ be the algebra of function classes on $O(H)$ constructed above. Then $\mathfrak{A}$ admits a unique expectation function $E$ which is invariant under the action $L_{(\cdot)}$ of $O(H)$ on $\mathfrak{A}$ where $L_{S} f(T)=f\left(S^{-1} T\right)$. The expectation $E$ is then invariant under the action $R_{(\cdot)}$ of $O(H)$, where $R_{S} f(T)=f(T S)$. 


\section{BiBLIOGRAPHY}

1. I. E. Segal, Tensor algebras over Hilbert spaces. II, Ann. of Math. (2) 63 (1965), 160-175

2. - Algebraic integration theory, Bull. Amer. Math. Soc. 71 (1965), 419-489.

3. - Explicit formal construction of non-linear quantum fields, J. Math. and Phys. 5 (1964), 269-282.

4. D. Shale and W. F. Stinespring, Integration over non-Euclidean geometries of infinite dimension, J. Math. Mech. (to appear).

University of Pennsylvania,

Philadel phia, Pennsylvania 